\title{
ON THE HAMILTONIAN FORMULATION OF NONHOLONOMIC MECHANICAL SYSTEMS
}

\author{
A. J. VAN DER SCHAFT \\ Dept. of Applied Mathematics, University of Twente, P.O. Box 2177500 AE Enschede, The Netherlands \\ and \\ B. M. MASCHKE \\ Lab. d'Automatique, Conservatoire National des Arts et Metiers, 21 Rue Pinel, 75013 Paris, France \\ (Received Februan 11, 1944 - Revised May 4, 1994))

\begin{abstract}
A simple procedure is provided to write the equations of motion of mechanical systems with constraints as Hamiltonian equations with respect to a "Poisson" bracket on the constrained state space, which does not necessarily satisfy the Jacobi identity. It is shown that the Jacobi identity is satisfied if and only if the constraints are holonomic.
\end{abstract}

\section{Introduction}

The theory of mechanical systems with nonholonomic constraints has a long history in classical mechanics; see e.g. the books by Neimark \& Fufaev [14], Edelen [6], Rosenberg [16], Arnold [1] and the references quoted in there. In this literature, nonholonomic mechanical systems are described within the variational framework by Euler-Lagrange equations with extra terms corresponding to the constraint forces.

The present note is largely influenced by a recent paper of Bates \& Śniatycki [4], see also Stanchenko [17], where it is shown that the dynamics of mechanical systems with nonholonomic constraints may be alternatively described within a $\mathrm{Ha}$ miltonian framework. However, the two-form with respect to which the Hamiltonian equations of motion (on a reduced state space, and without constraint forces) are defined is not necessarily closed, as may be demonstrated on simple examples. As a consequence, the resulting equations of motion, albeit of a Hamiltonian format, need not admit canonical coordinates and thus need not be transformable to the standard Hamiltonian equations: $\dot{q}_{i}=\frac{\partial H}{\partial p_{i}}, \dot{p}_{i}=-\frac{\partial H}{\partial q_{i}}, i=1, \ldots, n$. In the present note we will use, instead of the notion of a (not necessarily closed) two-form, the dual object of a "Poisson" bracket not necessarily satisfying the Jacobi identity. We will show in a simple manner that the dynamics of mechanical systems with holonomic or nonholonomic constraints is Hamiltonian with respect to such a generalized bracket. An explicit expression for this bracket is provided. Furthermore, we will show that this 
generalized bracket satisfies the Jacobi identity (and thus is a true Poisson bracket) if and only if the constraints are holonomic.

A second motivation for the developments in the present note comes from our previous work on the mathematical modelling of non-resistive physical systems (see [11], [12]). Here it has been shown that the network modelling of such systems immediately leads to a Hamiltonian dynamics with respect to a generalized bracket. In fact, the Hamiltonian function is given as the total stored energy, while the bracket is determined by the network topology (or junction structure). From a modelling point of view there is no clear a priori reason why this bracket should satisfy the Jacobi identity (although it does in many cases); and indeed the present note shows that for nonholonomic mechanical systems the Jacobi identity is never satisfied.

In this paper, we treat only the case of classical (kinematic) constraints, i.e. linear restrictions on the velocities. However it is clear, especially from the above mentioned modelling point of view, that more general constraints, as well as interconnections of systems, may be considered in the same framework. This and the control-theoretic implications (see e.g. [5], [3]) will be explored in a future paper.

Finally, an important motivation for the Hamiltonian formulation of nonholonomic dynamics in [4] is the treatment of symmetry and reduction for these systems. This will not be considered in the present note. For related work on the reduction of nonholonomic mechanical systems with symmetries from the Lagrangian point of view we refer to e.g. [7], [10], [2].

\section{The Hamiltonian formulation of systems with classical constraints}

Let $Q$ be an $n$-dimensional configuration manifold with local coordinates $q$ $=\left(q_{1}, \ldots, q_{n}\right)$. Consider a smooth Lagrangian function $L: T Q \rightarrow \mathbb{R}$, denoted by $L(q, \dot{q})$, satisfying throughout the usual regularity condition

$$
\operatorname{det}\left[\frac{\partial^{2} L}{\partial q_{i} \partial q_{j}}\right] \neq 0 .
$$

(This is e.g. satisfied if $L$ is given as the kinetic energy with positive definite generalized mass matrix minus the potential energy.) Classical constraints are given in local coordinates as

$$
A^{T}(q) \dot{q}=0,
$$

with $A(q)$ a $k \times n$ matrix, $k \leq n$, with entries depending smoothly on $q$. Throughout we assume that $A(q)$ has rank equal to $k$ everywhere. More intrinsically, the columns of $A(q)$ define in local coordinates $k$ independent one-forms on $Q$. Furthermore, (2) determines a $k$-dimensional distribution $D$ on $Q$, given in every point $q_{0} \in Q$ as

$$
D\left(q_{0}\right)=\operatorname{ker} A^{T}\left(q_{0}\right) .
$$

The constraints (2) are called holonomic if the distribution $D$ is involutive, i.e. for any two vectorfields $X, Y$ on $Q$

$$
X \in D, \quad Y \in D \Rightarrow[X, Y] \in D,
$$


with $[X, Y]$ the Lie bracket, defined in local coordinates $q$ as $[X, Y](q)=\frac{\partial Y}{\partial q}(q) X(q)-$ $-\frac{\partial X}{\partial q}(q) Y(q)$, with $\frac{\partial Y}{\partial y}, \frac{\partial X}{\partial q}$ the Jacobian matrices. In this case we may find, by Frobenius theorem, local coordinates $\bar{q}=\left(\bar{q}_{1}, \ldots, \bar{q}_{n}\right)$ such that the constraints (2) are expressed as

$$
\dot{\bar{q}}_{n-k+1}=\ldots=\dot{\bar{q}}_{n}=0,
$$

or equivalently, $\bar{q}_{n-k+1}=c_{n-k+1}, \ldots, \bar{q}_{n}=c_{n}$ for certain constants $c_{n-k+1}, \ldots, c_{n}$ determined by the initial conditions, and we may thus eliminate the coordinates $\bar{q}_{n-k+1}, \ldots, \bar{q}_{n}$. The constraints (2) are called nonholonomic if $D$ is not involutive, implying that we cannot use this elimination procedure.

The equations of motion for the mechanical system on $Q$ with Lagrangian $L(q, \dot{q})$ and constraints (2) are given as (see e.g. [14], [16], [1])

$$
\frac{d}{d t}\left(\frac{\partial L}{\partial \dot{q}}\right)-\frac{\partial L}{\partial q}=A(q) \lambda, \quad A^{T}(q) \dot{q}=0
$$

with $\frac{\partial L}{\partial q}$ denoting the column vector $\left(\frac{\partial L}{\partial q_{1}}, \ldots, \frac{\partial L}{\partial q_{n}}\right)^{T}$, and similarly for $\frac{\partial L}{\partial \dot{q}}$. Here the constraint forces $A(q(t)) \lambda(t)$ with $\lambda(t) \in \mathbb{R}^{k}$ are uniquely determined by the requirement that the constraints $A^{T}(q(t)) \dot{q}(t)=0$ have to be satisfied for all $t$.

Defining in the usual way the Hamiltonian $H(q, p)$ by the Legendre transformation

$$
H(q, p)=\sum_{i=1}^{n} p_{i} \dot{q}_{i}-L(q, \dot{q}), \quad p_{i}=\frac{\partial L}{\partial \dot{q}_{i}}, \quad i=1, \ldots, n,
$$

the constrained Euler-Lagrange equations (6) transform, due to condition (1), into the constrained Hamiltonian equations on $T^{*} Q$

$$
\begin{gathered}
\dot{q}=\frac{\partial H}{\partial p}(q, p), \quad p=-\frac{\partial H}{\partial q}(q, p)+A(q) \lambda, \\
A^{T}(q) \frac{\partial H}{\partial p}(q, p)=A^{T}(q) \dot{q}=0 .
\end{gathered}
$$

The constraint forces $A(q) \lambda$ may be computed by differentiating the constraints $A^{T}(q) \frac{\partial H}{\partial p}(q, p)=0$ along $(8)$, i.e.

$$
\left[\frac{\partial}{\partial q}\left(A^{T}(q) \frac{\partial H}{\partial p}(q, p)\right]^{T} \frac{\partial H}{\partial p}(q, p)+A^{T}(q) \frac{\partial^{2} H}{\partial p^{2}}(q, p)\left[-\frac{\partial H}{\partial q}(q, p)+A(q) \lambda\right]=0,\right.
$$

with $\frac{\partial^{2} H}{\partial p^{2}}$ the Hessian matrix with respect to $p$. This equation may be solved for $\lambda$ as long as

$$
\operatorname{det} A^{T}(q) \frac{\partial^{2} H}{\partial p^{2}}(q, p) A(q) \neq 0, \quad q \in Q,
$$

which condition is obviously satisfied because of our standing assumptions (1) and rank $A(q)=k$. Expressing $\lambda$ as a function of $(q, p)$ and substituting in (8) lead to 
the dynamical equations of motion on the constrained state space

$$
\mathcal{X}_{r}=\left\{(q, p) \in T^{*} Q \mid A^{T}(q) \frac{\partial H}{\partial p}(q, p)=0\right\} .
$$

A much more intrinsic and efficient way of obtaining the equations on $\mathcal{X}_{r}$ is, however, the following. Since rank $A(q)=k$, there exists locally a smooth $n \times(n-k)$ matrix $S(q)$ of rank $n-k$ such that

$$
A^{T}(q) S(q)=0 .
$$

(Equivalently, $S(q)$ is such that $D(q)=\operatorname{Im} S(q)$.) Now definc $\widetilde{p}=\left(\widetilde{p}^{1}, \widetilde{p}^{2}\right)=\left(\widetilde{p}_{1}, \ldots\right.$ $\left.\ldots, \widetilde{p}_{n-k}, \widetilde{p}_{n-k+1}, \ldots, \widetilde{p}_{n}\right)$ as

$$
\widetilde{p}^{1}:=S^{T}(q) p, \quad \widetilde{p}^{1} \in \mathbb{R}^{n-k}, \quad \widetilde{p}^{2}:=A^{T}(q) p, \quad \widetilde{p}^{2} \in \mathbb{R}^{k} .
$$

It immediately follows from (12) that $(q, p) \mapsto\left(q, \widetilde{p}^{1}, \widetilde{p}^{2}\right)$ is a coordinate transformation. The constrained Hamiltonian dynamics (8) in the ncw (gencrally not canonical!) coordinates $\left(q, \widetilde{p}^{1}, \widetilde{p}^{2}\right)$ takes the following form. The cotangent bundle $T^{*} Q$ is equipped with its canonical Poisson bracket expressed in natural canonical coordinates $(q, p)=\left(q_{1}, \ldots, q_{n}, p_{1}, \ldots, p_{n}\right)$ for $T^{*} Q$ as

$$
\begin{gathered}
\{F, G\}(q, p)=\sum_{i=1}^{n}\left(\frac{\partial F}{\partial q_{i}} \frac{\partial G}{\partial p_{i}}-\frac{\partial F}{\partial p_{i}} \frac{\partial G}{\partial q_{i}}\right)(q, p)=\left(\frac{\partial F^{T}}{\partial q} \frac{\partial F^{T}}{\partial p}\right) J\left(\begin{array}{c}
\frac{\partial G}{\partial q} \\
\frac{\partial G}{\partial p}
\end{array}\right), \\
J=\left(\begin{array}{cc}
O_{n} & I_{n} \\
-I_{n} & O_{n}
\end{array}\right),
\end{gathered}
$$

with $J$ the standard Poisson structure matrix (see e.g. [8]), intrinsically determined by the Poisson bracket $\{$,$\} as$

$$
J=\left(\begin{array}{ll}
\left(\left\{q_{i}, q_{j}\right\}\right)_{i, j} & \left(\left\{q_{i}, p_{j}\right\}\right)_{i, j} \\
\left(\left\{p_{i}, q_{j}\right\}\right)_{i, j} & \left(\left\{p_{i}, p_{j}\right\}\right)_{i, j}
\end{array}\right), \quad i, j=1, \ldots, n .
$$

In the new coordinates $(q, \tilde{p})$ the Poisson structure matrix becomes

$$
\widetilde{J}(q, \tilde{p})=\left(\begin{array}{cc}
\left(\left\{q_{i}, q_{j}\right\}\right)_{i, j} & \left(\left\{q_{i} \widetilde{p}_{j}\right\}\right)_{i, j} \\
\left(\left\{\widetilde{p}_{j}, q_{i}\right\}\right)_{i, j} & \left(\left\{\widetilde{p}_{i}, \widetilde{p}_{j}\right\}\right)_{i, j}
\end{array}\right), \quad i, j=1, \ldots, n,
$$

and the constrained Hamiltonian dynamics (8), equivalently written as

$$
\left(\begin{array}{l}
q \\
p
\end{array}\right)=J\left(\begin{array}{c}
\frac{\partial H}{\partial q}(q, p) \\
\frac{\partial H}{\partial p}(q, p)
\end{array}\right)+\left(\begin{array}{c}
0 \\
A(q)
\end{array}\right) \lambda, \quad A^{T}(q) \frac{\partial H}{\partial p}(q, p)=0
$$

transforms into 


$$
\left[\begin{array}{c}
\dot{q} \\
\dot{\tilde{p}}^{1} \\
\dot{\tilde{p}}^{2}
\end{array}\right]=\widetilde{J}(q, \widetilde{p})\left[\begin{array}{c}
\frac{\partial \widetilde{H}}{\partial q} \\
\frac{\partial \widetilde{H}}{\partial \widetilde{p}^{1}} \\
\frac{\partial \widetilde{H}}{\partial \widetilde{p}^{2}}
\end{array}\right]+\left[\begin{array}{c}
0 \\
0 \\
K(q)
\end{array}\right] \lambda, \quad \frac{\partial \widetilde{H}}{\partial \widetilde{p}^{2}}(q, \widetilde{p})=0,
$$

with $K(q):=A^{T}(q) A(q)$ an invertible $k \times k$ matrix, and $\widetilde{H}(q, \widetilde{p})$ the Hamiltonian $H(q, p)$ expressed in the new coordinates $q, \widetilde{p}$. Now truncate the transformed Poisson structure matrix $\widetilde{J}$ in (16) by leaving out the last $k$ columns and last $k$ rows, and let $\widetilde{p}$ satisfy the constraint equation $\frac{\partial \widetilde{H}}{\partial \widetilde{p}^{2}}=0$. This defines a $(2 n-k) \times$ $(2 n-k)$ skew-symmetric matrix $J_{r}$ on $\mathcal{X}_{r}$. An explicit expression for $J_{r}$ is obtained as follows. Denote the $j$-th column of $S(q)$ by $S_{j}(q)$. Since $\widetilde{p}^{1}=S^{T}(q) p$, we obtain $\left\{q_{l}, \tilde{p}_{j}\right\}=l$-th element of $S_{j}(q)$, while $\left\{\tilde{p}_{i}, \widetilde{p}_{j}\right\}=\left\{S_{i}^{T}(q) p, S_{j}^{T}(q) p\right\}=$ $-p^{T}\left[S_{i}, S_{j}\right](q), l=1, \ldots, n, i, j=1, \ldots, n-k$. Clearly $\left\{q_{i}, q_{j}\right\}=0$, and thus

$$
J_{r}=\left(\begin{array}{cc}
O_{n} & S(q) \\
-S^{T}(q) & \left(-p^{T}\left[S_{i}, S_{j}\right](q)\right)_{i, j=1, \ldots, n-k}
\end{array}\right),
$$

where $p$ is expressed as a function of $q, \widetilde{p}$ with $\widetilde{p}$ satisfying $\frac{\partial \widetilde{H}}{\partial \widetilde{p}^{2}}=0$. Note that rank $J_{r}=2(n-k)$ everywhere on $\mathcal{X}_{r}$. Furthermore, define the reduced Hamiltonian $H_{r}: \mathcal{X}_{r} \rightarrow \mathbb{R}$ as $\widetilde{H}(q, \widetilde{p})$ with $\widetilde{p}$ satisfying $\frac{\partial \widetilde{H}}{\partial \widetilde{p}^{2}}=0$. Clearly, $\left(q, \widetilde{p}^{1}\right)$ serve as local coordinates for the constrained state space $\mathcal{X}_{r}$. It immediately follows from (18) by disregarding the last equations involving $\lambda$, and noting that $\frac{\partial \widetilde{H}}{\partial \widetilde{p}^{2}}(q, \widetilde{p})=0$, that the dynamical equations on $\mathcal{X}_{r}$ expressed in coordinates $\left(q, \widetilde{p}^{1}\right)$ are given as

$$
\left(\begin{array}{c}
\dot{q} \\
\dot{\tilde{p}}^{1}
\end{array}\right)=J_{r}\left(q, \widetilde{p}^{1}\right)\left(\begin{array}{c}
\frac{\partial H_{r}}{\partial q}\left(q, \widetilde{p}^{1}\right) \\
\frac{\partial H_{r}}{\partial \widetilde{p}^{1}}\left(q, \tilde{p}^{1}\right)
\end{array}\right), \quad\left(\begin{array}{c}
q \\
\widetilde{p}^{1}
\end{array}\right) \in \mathcal{X}_{r} .
$$

These equations are in pseudo-Hamiltonian format. Indeed, the matrix $J_{r}$ defines a bracket $\{,\}_{r}$ on $\mathcal{X}_{r}$ by setting

$$
\left\{F_{r}, G_{r}\right\}_{r}\left(q, \widetilde{p}^{1}\right):=\left(\frac{\partial F_{r}^{T}}{\partial q} \frac{\partial F_{r}^{T}}{\partial \widetilde{p}^{1}}\right) J_{r}\left(q, \widetilde{p}^{1}\right)\left(\begin{array}{c}
\frac{\partial G_{r}}{\partial q} \\
\frac{\partial G_{r}}{\partial \widetilde{p}^{1}}
\end{array}\right)
$$

for any two smooth functions $F_{r}, G_{r}: \mathcal{X}_{r} \rightarrow \mathbb{R}$. Clearly, this bracket satisfies the first two defining properties of a Poisson bracket (see e.g. [8], [15], [9]):

(i) $\left\{F_{r}, G_{r}\right\}_{r}=-\left\{G_{r}, F_{r}\right\}_{r}$ (skew-symmetry),

(ii) $\left\{F_{r}, G_{r}, H_{r}\right\}_{r}=\left\{F_{r}, G_{r}\right\}_{r} H_{r}+G_{r}\left\{F_{r}, H_{r}\right\}_{r}$ (Leibniz rule) 
for every $F_{r}, G_{r}, H_{r}: \mathcal{X}_{r} \rightarrow \mathbb{R}$. However, for $\{,\}_{r}$ to be a true Poisson bracket also the following property:

(iii) $\left\{F_{r},\left\{G_{r}, H_{r}\right\}_{r}\right\}_{r}+\left\{G_{r},\left\{H_{r}, F_{r}\right\}_{r}\right\}_{r}+\left\{H_{r},\left\{F_{r}, G_{r}\right\}_{r}\right\}_{r}=0$

$$
\text { (Jacobi identity) }
$$

needs to be satisfied. If (23) is satisfied, then (20) defines a generalized Hamiltonian system on $\mathcal{X}_{r}$ with respect to the Poisson bracket $J_{r}$ (see e.g. [15], [9], [11]), and since rank $J_{r}=2(n-k)$ local (canonical) coordinates $(\bar{q}, \bar{p}, \bar{s})$ for $\mathcal{X}_{r}$ may be found such that the system takes the form $[8,15,11]$

$$
\dot{\bar{q}}=\frac{\partial H_{r}}{\partial \bar{p}}, \quad \dot{\bar{p}}=-\frac{\partial H_{r}}{\partial \bar{q}}, \quad \dot{\bar{s}}=0, \quad \bar{q}, \bar{p} \in \mathbb{R}^{n-k}, \quad \bar{s} \in \mathbb{R}^{k}
$$

(with $H_{r}$ expressed in $\bar{q}, \bar{p}, \bar{s}$ ), which are almost the standard Hamiltonian equations of motion. On the other hand, even if (23) is not satisfied, then still the pseudo-Hamiltonian format may be useful, see e.g. Remark 2.2 and the Conclusions.

The next theorem shows that the fulfilment of the Jacobi identity is actually equivalent to the constraints being holonomic.

THEOREM 2.1. The bracket $\{,\}_{r}$ on $\mathcal{X}_{r}$ satisfies the Jacobi identity (and thus is a Poisson bracket) if and only if the constraints $A^{T}(q) \dot{q}=0$ are holonomic.

Proof: (If) Suppose the constraints are holonomic. Then by Frobenius' theorem we may find local coordinates $\bar{q}=\left(\bar{q}_{1}, \ldots, \bar{q}_{n}\right)$ such that the constraints are expressed as in (5), i.e. $A^{T}(\bar{q})=\left[\begin{array}{ll}0 & I_{k}\end{array}\right]$. Take corresponding (natural) coordinates $(\bar{q}, \bar{p})$ for $T^{*} Q$, and denote $\bar{p}=\left(\bar{p}^{1}, \bar{p}^{2}\right)=\left(\bar{p}_{1}, \ldots, \bar{p}_{n-k}, \bar{p}_{n-k+1}, \ldots, \bar{p}_{n}\right)$. The constraint is now expressed as $\frac{\partial H}{\partial \widetilde{p}^{2}}(\bar{q}, \bar{p})=0$, and we can take as in (13) new coordinates $\widetilde{p}^{1}=\bar{p}^{1}, \widetilde{p}^{2}=$ $\bar{p}^{2}$. Since in these coordinates $J=\left(\begin{array}{cc}0 & I_{n} \\ -I_{n} & 0\end{array}\right), J_{r}$ becomes the constant matrix

$$
\widetilde{\jmath}=\left[\begin{array}{ccc}
0_{n} & & I_{n-k} \\
& & 0 \\
-I_{n-k} & 0 & 0
\end{array}\right],
$$

and $\{,\}_{r}$ trivially satisfies the Jacobi identity.

(Only if) Suppose the bracket $\{,\}_{r}$ defined by $J_{r}$ satisfies the Jacobi identity. Denote the Hamiltonian vectorfields on $\mathcal{X}_{r}$ (with respect to $J_{r}$ ) with Hamiltonians $q_{1}, \ldots, q_{n}, \widetilde{p}_{1}, \ldots, \widetilde{p}_{n-k}$ by $X_{q_{1}}, \ldots, X_{q_{n}}, X_{\tilde{p}_{1}}, \ldots, X_{\tilde{p}_{n-k}}$. By definition, these are precisely the columns of $J_{r}$. Fulfilment of the Jacobi identity implies that (see e.g. [8])

$$
\left[X_{\tilde{p}_{i}}, X_{\tilde{p}_{j}}\right]=X_{\left\{\tilde{p}_{i}, \tilde{p}_{j}\right\}_{r}}, \quad i, j=1, \ldots, n-k .
$$

In particular, this implies that $\left[X_{\tilde{p}_{i}}, X_{\hat{p}_{j}}\right]$ is in the span of the columns of $J_{r}$. However, since by (19) the vector of first $n$ entries of $X_{\tilde{p}_{i}}$ is precisely the $i$-th column of $S(q)$, this implies by the occurrence of the left-upper zero block $0_{n}$ in $J_{r}$ that $\left[S_{i}, S_{j}\right](q) \in \operatorname{Im} S(q)$, with $S_{i}$ the $i$-th column of $S, i, j=1, \ldots, n-k$. Hence, the distribution $D(q)=\operatorname{Im} S(q)$ is involutive. 
Remark 2.2: In any case (Jacobi identity being satisfied or not) the dynamics (20) is energy conserving, i.e., $\widetilde{H}_{r}$ remains constant along trajectories of (20), as follows from skew-symmetry of $J_{r}$. This may be e.g. used for feedback stabilization of nonholonomic systems [13].

Remark 2.3: The dynamics (20) on $\mathcal{X}_{r}$ is independent of the particular choice of coordinates for $\mathcal{X}_{r}$, as may be readily checked.

Remark 2.4: Our approach is not unrelated to the approach taken in [5]. Here the multipliers $\lambda$ in the Euler-Lagrange equations (6) are eliminated by pre-multiplying the equations (6) by the matrix $S^{T}(q)$, and it is shown that the thus reduced equations can be written as a set of first-order differential equations in $q$ and $\eta \in \mathbb{R}^{n-k}$, with $\dot{q}=S(q) \eta$ parametrizing the admissible velocities $\dot{q}$.

We now treat two well-known examples of nonholonomic mechanical systems; the first one has been also considered within a (different) Hamiltonian framework in [4].

EXAMPLE 2.5 (see e.g. [16]). Consider the motion of a point mass $m$ in $Q=\mathbb{R}^{3}$ with Cartesian coordinates $(x, y, z)$ under the influence of a potential field with potential $V(x, y, z)$, and subject to the nonholonomic constraint $\dot{z}=y \dot{x}$. The constrained Hamiltonian equations (8) are given as

$$
\begin{array}{ll}
\dot{x}=\frac{1}{m} p_{x}, & \dot{p}_{x}=-\frac{\partial V}{\partial x}-y \cdot \lambda, \\
\dot{y}=\frac{1}{m} p_{y}, & \dot{p}_{y}=-\frac{\partial V}{\partial y}, \quad p_{z}-y p_{x}=0, \\
\dot{z}=\frac{1}{m} p_{z}, & \dot{p}_{z}=-\frac{\partial V}{\partial z}+\lambda .
\end{array}
$$

Define, corresponding to (13), the new coordinates

$$
\widetilde{p}_{x}=p_{x}+y p_{z}, \quad \widetilde{p}_{y}=p_{y}, \quad \widetilde{p}_{z}=p_{z}-y p_{x},
$$

then the Poisson structure matrix $J=\left(\begin{array}{cc}0 & I_{3} \\ -I_{3} & 0\end{array}\right)$ transforms into

$$
\widetilde{J}=\left[\begin{array}{rrrccc}
0 & 0 & 0 & 1 & 0 & -y \\
0 & 0 & 0 & 0 & 1 & 0 \\
0 & 0 & 0 & y & 0 & 1 \\
-1 & 0 & -y & 0 & p_{z} & 0 \\
0 & -1 & 0 & -p_{z} & 0 & p_{x} \\
y & 0 & -1 & 0 & -p_{x} & 0
\end{array}\right]
$$

with $p_{x}, p_{z}$ expressed in the new coordinates. The reduced structure matrix on $\mathcal{X}_{r}=$ $\left.\left\{x, y, z, p_{x}, p_{y}, p_{z}\right) \mid \tilde{p}_{z}=0\right\}$ becomes (note that on $\mathcal{X}_{r}, p_{x}=\widetilde{p}_{x}\left(1+y^{2}\right), p_{z}=y \widetilde{p}_{x} /\left(1+y^{2}\right)$ ) 


$$
J_{r}=\left[\begin{array}{ccccc}
0 & 0 & 0 & 1 & 0 \\
0 & 0 & 0 & 0 & 1 \\
0 & 0 & 0 & y & 0 \\
-1 & 0 & -y & 0 & \frac{y \widetilde{p}_{x}}{1+y^{2}} \\
0 & -1 & 0 & -\frac{y \widetilde{p}_{x}}{1+y^{2}} & 0
\end{array}\right],
$$

which indeed does not satisfy the Jacobi identity. The reduced Hamiltonian $H_{r}$ is given as

$$
H_{r}\left(x, y, z, p_{x}, p_{y}\right)=\frac{1}{2 m}\left(\frac{1}{1+y^{2}} p_{x}^{2}+p_{y}^{2}\right)+V(x, y, z)
$$

and the resulting equations are described by (20) with $q=(x, y, z), \widetilde{p}^{1}=\left(p_{x}, p_{y}\right)$.

EXAMPLE 2.6 (Rolling vertical wheel on inclined plane, see e.g. [3].) The configuration manifold is $\mathbb{R}^{2} \times S^{1} \times S^{1}$ with coordinates $(x, y, \theta, \varphi)$; i.e. $(x, y)$ Cartesian coordinates on the plane, $\theta$ rotation angle of the wheel, and $\varphi$ heading angle. For simplicity we take all parameters to be 1 , leading to the Hamiltonian $H=\frac{1}{2}\left(p_{x}^{2}+p_{y}^{2}+p_{\theta}^{2}+p_{\varphi}^{2}\right)+x$. The rolling constraints are nonholonomic and given by $\dot{x}-\dot{\theta} \cos \varphi=0, \dot{y}-\dot{\theta} \sin \varphi=0$. According to (13), we define new coordinates

$$
\begin{aligned}
& \widetilde{p}_{1}:=p_{\varphi}, \\
& \widetilde{p}_{2}:=p_{\theta}+p_{x} \cos \varphi+p_{y} \sin \varphi, \\
& \widetilde{p}_{3}:=p_{x}-p_{\theta} \cos \varphi, \\
& \widetilde{p}_{4}:=p_{y}-p_{\theta} \sin \varphi .
\end{aligned}
$$

Then, $\mathcal{X}_{r}=\left\{\left(x, y, \theta, \varphi, p_{x}, p_{y}, p_{\theta}, p_{\varphi}\right) \mid \tilde{p}_{3}=0, \tilde{p}_{4}=0\right\}$, and the structure matrix $J_{r}$ may be computed as

$$
J_{r}=\left[\begin{array}{ccrrcc}
0 & 0 & 0 & 0 & 0 & \cos \varphi \\
0 & 0 & 0 & 0 & 0 & \sin \varphi \\
0 & 0 & 0 & 0 & 0 & 1 \\
0 & 0 & 0 & 0 & 1 & 0 \\
0 & 0 & 0 & -1 & 0 & 0 \\
-\cos \varphi & -\sin \varphi & -1 & 0 & 0 & 0
\end{array}\right]
$$

while the reduced Hamiltonian is given as $\widetilde{H}\left(x, y, \theta, \varphi, \widetilde{p}_{1}, \widetilde{p}_{2}\right)=\frac{1}{2} \widetilde{p}_{1}^{2}+\frac{1}{4} \widetilde{p}_{2}^{2}+x$.

\section{Conclusions}

We have shown that the equations of motion of a mechanical system with constraints may be easily formulated as Hamiltonian equations of motion with respect to a Hamiltonian which equals the total energy restricted to the constrained state space, and with respect to a bracket which satisfies the Jacobi identity if and only if the constraints are holonomic. This underlines the difficulties of nonholonomic constraints, and also motivates a further study of brackets not satisfying the Jacobi identity and 
their Hamiltonian equations of motion. It should be noted, however, that many useful results of Hamiltonian vectorfields, such as the existence of canonical coordinates and Liouville's theorem (conservation of volume) rely crucially on the fact that the Poisson bracket does satisfy the Jacobi identity.

\section{REFERENCES}

[1] V. I. Arnold (ed): Dynamical Systems III (Encyclopaedia of Mathematical Sciences, Vol. 3), Springer, Berlin 1988 (translation of the Russian original, 1985).

[2] A. Bloch, P. S. Krishnaprasad, J. E. Marsden and R. Murray: Nonholonomic mechanics and reduction, in preparation.

[3] A. M. Bloch, M. Reyhanoglu and N. H. McClamroch: IEEE Trans. Aut. Control 37 (1992), 1746.

[4] L. Bates and J. Śniatycki: Rep. Math. Phys. 32 (1993), 99.

[5] G. Campion, B. d'Andrea-Novel and G. Bastin: Controllability and state feedback stabilisability of nonholonomic mechanical systems, in Advanced Robot Control (ed. C. Canudas de Wit), LNCIS 162, Springer, Berlin 1991, 106-124.

[6] D. G. B. Edelen: Lagrangian Mechanics of Nonconservative Nonholonomic Systems, Noordhoff, Leiden 1977.

[7] J. Koiler: Arch. Rat. Mech. Anal. 118 (1992), 113.

[8] P. Libermann and C. M. Marle: Symplectic Geometry and Analytical Mechanics, Reidel, Dordrecht 1987.

[9] J. E. Marsden: Lectures on Mechanics, London Mathematical Society Lecture Note Series 174, Cambridge University Press, Cambridge 1992.

[10] J. E. Marsden and J. Scheurle: Lagrangian reduction and the double spherical pendulum, preprint 1992.

[11] B. Maschke, A. J. van der Schaft and P. C. Breedveld: J. Franklin Institute 329 (1992), 923.

[12] B. Maschke, A. J. van der Schaft and P. C. Breedveld: An intrinsic Hamiltonian formulation of the dynamics of $L C$-circuits, to appear in IEEE Transaction on Circuits and Systems, 1993.

[13] B. Maschke and A. J. van der Schaft: A Hamiltonian approach to stabilization of nonholonomic mechanics systems, to appear in Proc. 33rd IEEE Conf. Decision and Control, Orlando 1994.

[14] Y. Neimark and N. A. Fufaev: Dynamics of Nonholonomic Systems, Amer. Math. Soc. Translations 33, 1972.

[15] P. J. Olver: Applications of Lie Groups to Differential Equations, Springer, New York 1986.

[16] Rosenberg: Analytical Dynamics, Plenum Press, New York 1977.

[17] S. V. Stanchenko: J. Appl. Math. \& Mechanics (Prikl. Matem. Mekhan.) 53 (1989), 11. 